\title{
Can Vaccine-induced Mucosal High Avidity CD8+ CTL Delay AIDS-viral Dissemination from Mucosa?
}

Igor M Belyakov¹, Vladimir A Kuznetsov², Brian Kelsall ${ }^{3}$, Dennis Klinman ${ }^{4}$, Marcin Moniuszko ${ }^{1}$, Michael Lemon ${ }^{1}$, Phillip D Markham ${ }^{5}$, Ranajit Pal5, John D Clements ${ }^{6}$, Mark G Lewis ${ }^{7}$, Warren Strober $^{3}$, Genoveffa Franchini ${ }^{1}$ and Jay A Berzofsky* $\neq 1$

\author{
Address: ${ }^{1}$ Vaccine Branch, NCI, NIAID, NIH, Bethesda, MD 20892, USA, ${ }^{2}$ Division of Information and Mathematical Sciences, Genome Institute \\ of Singapore, 138672, Singapore, ${ }^{3}$ Laboratory Of Clinical Investigation, NIAID, NIH, Bethesda, MD 20892, USA, ${ }^{4}$ Center for Biologics Evaluation \\ and Research, Food and Drug Administration, Bethesda, MD, 20892, USA, ${ }^{5}$ Advanced BioScience Laboratories, Inc., Kensington, MD 20895, USA, \\ ${ }^{6}$ Department of Microbiology and Immunology, Tulane University School of Medicine, New Orleans, LA 70112, USA and ${ }^{7}$ Southern Research \\ Institute, Frederick, MD, USA \\ Email: Jay A Berzofsky* - berzofsk@helix.nih.gov \\ * Corresponding author $\ddagger$ Presenting author
}

from 2005 International Meeting of The Institute of Human Virology

Baltimore, USA, 29 August - 2 September 2005

Published: 8 December 2005

Retrovirology 2005, 2(SuppI I):SI00 doi: I0.I I86/I742-4690-2-SI-SI 00

Natural HIV transmission occurs through mucosa, but it is debated whether mucosal cytotoxic T lymphocytes (CTL) can prevent or reduce dissemination from the initial mucosal site to the systemic circulation. Also, the role of CTL avidity in mucosal AIDS viral transmission is unknown. To address these questions, we used delay in acute-phase peak viremia after intrarectal challenge as an indicator of systemic dissemination. We find that a peptide-prime/poxviral boost vaccine inducing high levels of high avidity mucosal CTL can impact dissemination of intrarectally administered pathogenic SHIV-ku2 in macaques, and that such protection correlates better with mucosal than with systemic CTL and particularly with levels of high avidity mucosal CTL. 